\title{
Effect of oxygen permeability on corrosion properties in mortar specimens mixed with bacillus subtilis
}

\author{
Saaya Sugitani ${ }^{1}$, Keiyu Kawaai $^{1, *}$, and Isao Ujike $^{1}$ \\ ${ }^{1}$ Ehime University, Civil and Environmental Engineering, 3, Bunkyocho, Matsuyama, Ehime, Japan
}

\begin{abstract}
The effect of the degree of water saturation on oxygen permeability in mortar specimens mixed with an aerobic microorganism of bacillus subtilis (natto) under various water saturation conditions is explored in this study. In the experimental program an immersion test was conducted using distilled water and salt water $(10 \%)$ under room conditions controlled at a constant temperature of $20^{\circ} \mathrm{C}$. The oxygen permeability on the surface of steel bars embedded at different saturation conditions in mortar specimens were examined by electrochemical measurements e.g. half-cell potential measurements, cathodic polarization techniques. Based on the results obtained, the rate of oxygen permeability of the mortar specimens mixed with the bacillus subtilis (natto) was clearly reduced from the age of 28 days to 91 days. The humid environment is advantageous to the metabolic activity of bacillus subtilis (natto) which is likely to occur in saturated conditions compared to those in relatively dry environments. This led to more negative half-cell potentials in the mortar specimens mixed with the bacillus subtilis (natto) especially under the saturated conditions. The variations of half-cell potentials could be attributed to variations of cathodic polarization properties which is illustrated by decreased oxygen permeability in the specimens.
\end{abstract}

\section{Introduction}

Corrosion of steel bars in concrete proceeds in the presence of water and dissolved oxygen via electrochemical reactions. When a corrosion cell is formed on the steel bars in concrete owing to chloride attack or carbonation, iron metal is ionized at the anodic region and is oxidized to $\mathrm{Fe}^{2+}$ while oxygen is concomitantly reduced at the cathodic regions. The cathodic reactions require dissolved oxygen present in pore solution, thus suggesting that the oxygen permeability is a significant factor contributing to propagation of corrosion.

Ingress of oxygen into the depth of steel bars embedded in concrete is likely to occur through the processes of diffusion of oxygen gas in the dried pore structure, dissolution of oxygen gas into the pore solution, and diffusion of dissolved oxygen as shown in Fig.1. Kawaai et al., (2018) showed that the rate of oxygen permeability can be decreased owing to reduced availability of dissolved oxygen through the metabolic processes of bacillus subtilis (natto) in mortar specimens cast with a partial replacement of mixing water with culture solution. This led to higher corrosion resistance even if bending cracking occurs in the mortar specimens. The results suggest that there is a good possibility that microcell and macrocell corrosion current density are decreased, which could be attributed to the enhancement of resistance against the cathodic reactions. This study examines the effect of the degree of water saturation on the oxygen permeability in mortar specimens mixed with bacillus subtilis (natto). In particular, the difference of metabolic activity which can be affected by the
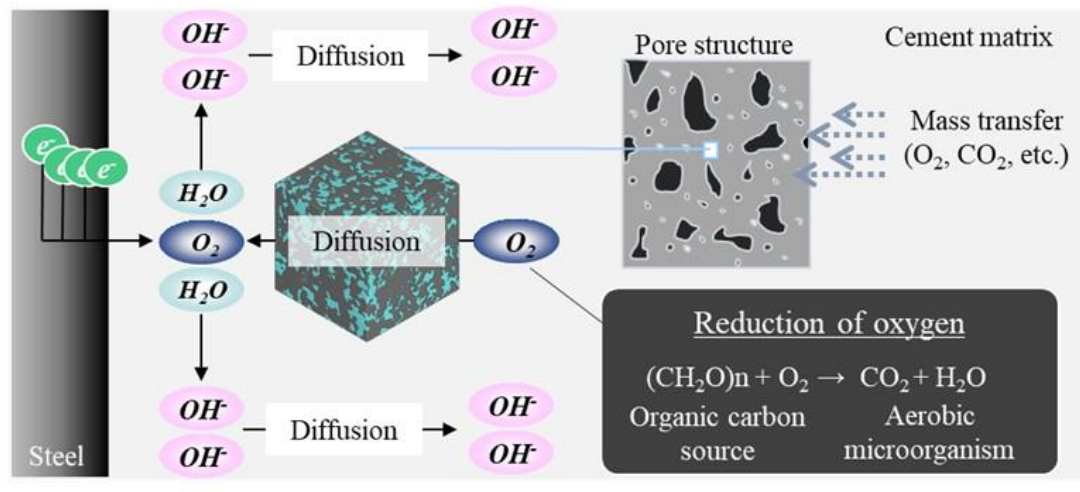

\section{Ingress of oxygen: \\ - Diffusion of oxygen gas \\ - Dissolution of oxygen gas into pore solution \\ - Diffusion of dissolved oxygen}

\section{Electro-chemical reactions}

Anode: $\mathrm{Fe} \rightarrow \mathrm{Fe}^{2+}+2 e^{-}$

Cathode: $2 \mathrm{H}_{2} \mathrm{O}+\mathrm{O}_{2}+4 e^{-} \rightarrow 4 \mathrm{OH}^{-}$

Reduction of cathodic reactions

Fig. 1. Schematic of oxygen permeability and cathodic reactions in corrosion processes

\footnotetext{
* Corresponding author: kkawaai@cee.ehime-u.ac.jp
} 
Table 1. Chemical composition of culture media.

\begin{tabular}{lr}
\hline \multicolumn{1}{c}{ Chemicals } & $\mathrm{g} / \mathrm{L}$ \\
\hline $\mathrm{NH}_{4} \mathrm{Cl}$ & 5 \\
$\mathrm{NH}_{4} \mathrm{NO}_{3}$ & 1 \\
$\mathrm{Na}_{2} \mathrm{SO}_{4}$ & 2 \\
$\mathrm{MgSO}_{4} \cdot 7 \mathrm{H}_{2} \mathrm{O}$ & 0.1 \\
$\mathrm{CaCl}_{2}$ & 1000 \\
$\mathrm{~K}_{2} \mathrm{HPO}_{4}$ & 3 \\
$\mathrm{KH}_{2} \mathrm{PO}_{4}$ & 1 \\
$\mathrm{C}_{6} \mathrm{H}_{12} \mathrm{O}_{6}$ & 0.5 \\
$\mathrm{H}_{2} \mathrm{O}$ & 1000 \\
$\mathrm{C}_{10} \mathrm{H}_{16} \mathrm{~N}_{2} \mathrm{O}_{3} \mathrm{~S}$ & 0.5 \\
Trace element & $1 \mathrm{~mL}$ \\
\hline
\end{tabular}

Table 2. Chemical composition of trace element.

\begin{tabular}{lr}
\hline \multicolumn{1}{c}{ Chemicals } & $\mathrm{mg} / \mathrm{mL}$ \\
\hline $\mathrm{Na}_{2} \mathrm{~B}_{4} \mathrm{O}_{7} \cdot 10 \mathrm{H}_{2} \mathrm{O}$ & 88 \\
$\mathrm{CuSO}_{4} \cdot 5 \mathrm{H}_{2} \mathrm{O}$ & 393 \\
$\mathrm{Fe}_{2}\left(\mathrm{SO}_{4}\right)_{3} \cdot 6 \mathrm{H}_{2} \mathrm{O}$ & 910 \\
$\mathrm{MnCl}_{2} \cdot 4 \mathrm{H}_{2} \mathrm{O}$ & 72 \\
$\left(\mathrm{NH}_{4}\right)_{6} \mathrm{Mo}_{7} \mathrm{O}_{24} \cdot 4 \mathrm{H}_{2} \mathrm{O}$ & 37 \\
$\mathrm{ZnSO}_{4} \cdot 7 \mathrm{H}_{2} \mathrm{O}$ & 8807 \\
$\mathrm{H}_{2} \mathrm{O}$ & 1000 \\
\hline
\end{tabular}

Table 3. Mix proportion.

\begin{tabular}{ccccccc}
\hline & \multicolumn{2}{c}{ Unit Weight kg/m } & & \multicolumn{2}{c}{ Unit Weight $\mathrm{g} / \mathrm{m}^{3}$} \\
\cline { 2 - 7 } Cases & $\mathrm{W}$ & $\mathrm{C}$ & $\mathrm{S}$ & $\mathrm{N} 1$ & AE-WRA & AE \\
& 278 & 505 & 1363 & 0 & 1668 & 556 \\
OPC & 209 & 505 & 1363 & 70 & 1668 & 556 \\
\hline
\end{tabular}

Note: N1 (bacillus subtilis) is mixed after cultivated for 24 hours. Mixing water (tap water) is replaced with a replacement ratio of 0.25 .

availability of water and dissolved oxygen is investigated using segmented steel bars embedded in mortar specimens under various wet/dry environments.

\section{Experimental programs}

This section provides detailed information about experimental programs carried out in this study. In particular, materials and testing methods associated with evaluation of oxygen permeability via electro-chemical measurements are described.

\subsection{Cultivation of bacillus subtilis (natto)}

The rod-shaped ( $1 \mu \mathrm{m}$ width and 2-3 $\mu \mathrm{m}$ length) bacillus subtilis (natto) is resistant to unfavourable environmental conditions including salinity and extreme $\mathrm{pH}$ through the formation of an endospore at times of nutritional stress until conditions become favourable. The application would be classified into two categories. An approach is that the bacillus subtilis (natto) is mixed in mixing water before concrete specimens are cast. If they could actively metabolize organic carbon sources provided, the dissolved oxygen concentration is reduced through the aerobic processes. The other approach is that a mixture containing the bacillus subtilis (natto) is sprayed into cracks in concrete. The application is very similar to the liquid-based repair techniques (Ujike et al., 2014; Wiktor and Jonkers, 2015). As mentioned in the introduction, it is clear that the bacillus subtilis (natto) could be compatible with the alkaline environment in pores or cracks saturated with higher $\mathrm{pH}$ solution.

The bacillus subtilis (natto) used in this study was cultivated using culture media which is typically used for minimal medium for E. coli. and the details of chemical composition are summarised in Tables 1 and 2. The mixing process should be started with the chemicals listed in the top of the table in order. They were diluted 100 times in mass using distilled water. For the cultivation procedure, nutrients and food such as an organic carbon source which was glucose $\left(\mathrm{C}_{6} \mathrm{H}_{12} \mathrm{O}_{6}\right)$ in this study should be provided and aeration apparatus was also utilized in the container.

\subsection{Mortar specimens}

Fig. 2 shows the schematic of prism mortar specimens used in this study. The specimens, of cross section $50 \mathrm{x}$ $50 \mathrm{~mm}$, length of $150 \mathrm{~mm}$ were cast with mortar mixtures with and without bacillus subtilis (natto). Segmented steel bars (20 mm each) comprising 3 steel elements which are connected by epoxy resin were embedded in the specimens. The cover depth was $20 \mathrm{~mm}$ and duplicate specimens were made to confirm the consistency of the results. It should be noted that the specimens were sealed by epoxy resin except for an exposed surface. Table 3 shows the mix proportions of the mortar mixtures used in this study. The cement used was high early strength Portland cement $\left(3.14 \mathrm{~g} / \mathrm{cm}^{3}\right)$ in accordance with JIS R 5210. The fine aggregate was crushed sand obtained from sandstone with specific 


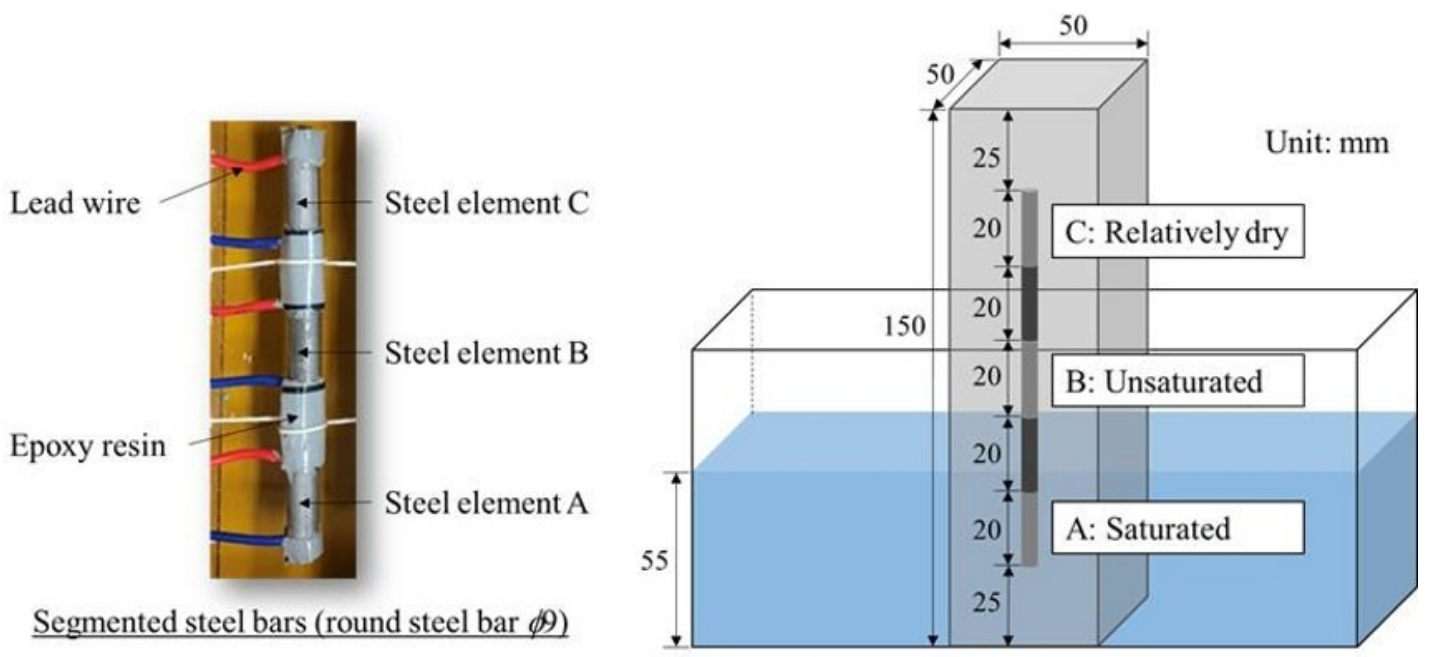

Fig. 2. Schematic of mortar specimen and segmented steel bars

gravity, water absorption, and fineness modulus of 2.61 $\mathrm{g} / \mathrm{cm}^{3}, 1.06 \%$, and 2.92 respectively. The OPC mixture was a control mixture and N25 was a mixture with culture solution of bacillus subtilis (natto) with a replacement ratio of 0.25 . The specimens were cured in a water tank until the age of 28 days immediately before the immersion test was started.

\subsection{Electro-chemical measurements}

Electro-chemical measurements were carried out via half-cell potential measurements, AC impedance method, and cathodic polarization measurements. The half-cell potential was measured in accordance with ACTM-C 876 from the concrete surface using a $\mathrm{Ag} / \mathrm{AgCl}$ reference electrode saturated with $\mathrm{KCl}$. The potentials based on a $\mathrm{Cu} / \mathrm{CuSO}_{4}$ electrode were estimated by the readings. The set-up for the oxygen permeability measurement is similar to polarization resistance measurements. The electro-chemical measurement by a potentiodynamic test comprised a working electrode (embedded steel bars), counter electrode (stainless steel plate), and a reference electrode $(\mathrm{Ag} / \mathrm{AgCl})$. When $\mathrm{DC}$ current was impressed by the rate of $1 \mathrm{mV} / \mathrm{s}$, the current density was measured by a potentiostat. It should be noted that no IR correction was made on the results in this study. The limiting current density was assumed when the potential difference reached $860 \mathrm{mV}$ and oxygen permeability in concrete specimens was calculated based on Eq. (1) (Nagataki et al., 1996).

$$
\frac{d Q}{d t}=-\frac{i_{l i m}}{n F}
$$

where $\mathrm{dQ} / \mathrm{dt}$ : the rate of oxygen permeability $\left(\mathrm{mol} / \mathrm{cm}^{2} / \mathrm{sec}\right) ; \mathrm{i}_{\text {lim }}$ : limiting current density $\left(\mathrm{A} / \mathrm{cm}^{2}\right) ; \mathrm{F}$ : Faradays constant $(96,500$ coulombs/mol); and $\mathrm{n}$ : the
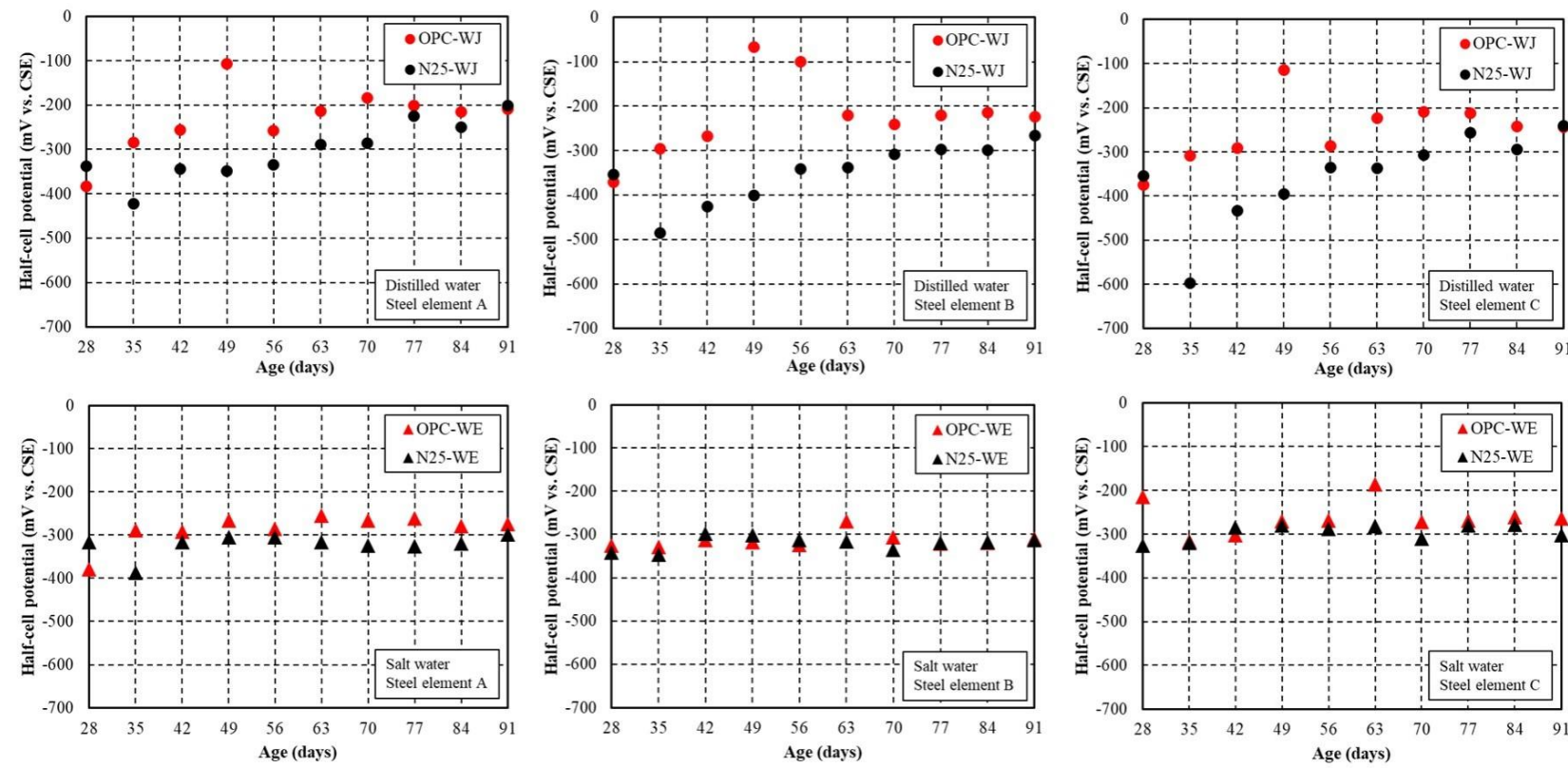

Fig. 3. Results of half-cell potential in the cases of steel elements A, B, and C in the OPC and N25 specimens 
number of electrons exchanged equal to 4 .

\section{Results and discussion}

This section reports on the results of immersion tests carried out on the mortar specimens. Variations of halfcell potential and oxygen permeability measured by cathodic polarization techniques under various saturation degree are central to this study.

\subsection{Results of half-cell potential and corrosion current density}

Fig. 3 shows results of half-cell potentials measured on the steel elements $\mathrm{A}, \mathrm{B}$ and $\mathrm{C}$ after being exposed to the immersion tests. As can be seen, the half-cell potentials of steel elements $\mathrm{A}, \mathrm{B}$, and $\mathrm{C}$ in the $\mathrm{N} 25-\mathrm{WJ}$ were observed to be lower compared to those observed in the OPC-WJ. They were clearly lower than $-350 \mathrm{mV}$ vs. CSE which is a threshold value indicating that possibility of corrosion is greater than $90 \%$. The difference with regards to potentials between OPC-WJ and N25-WJ mixtures was significant until the age of 91 days in this study. On the other hand, the potentials measured in the OPC-WE and N25-WE mixtures were comparable, which ranged from -200 to $-400 \mathrm{mV}$ vs. CSE. The typical ranges of potentials of normal steel in concrete are reported by RILEM technical commetee (2003). The potentials measured in water saturated concrete without oxygen generally show more negative values compared to those of chloride contaminated concrete. The effect of the addition of the bacillus subtilis (natto) under the saturated condition (steel element A) is subsequently discussed.

The potential difference between OPC-WJ and N25WJ was calculated until the age of 182 days as shown in Fig. 4. It should be noted that the half-cell potential greater than $-200 \mathrm{mV}$ vs. CSE in the OPC-WJ is considered to be consistently $-200 \mathrm{mV}$ taking into consideration the variations of potential measurements. When the values calculated based on the potential

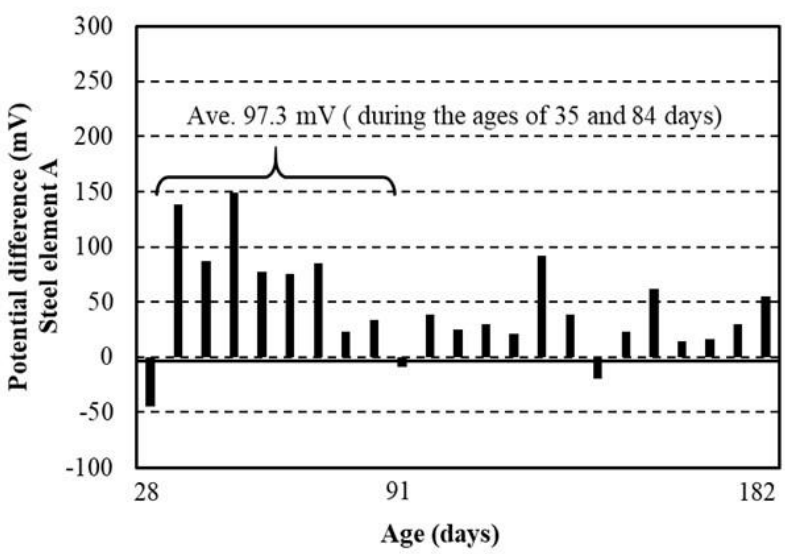

Fig. 4. Potential difference (OPC-WJ and N25WJ)

difference are positive, this indicates that the potential of $\mathrm{N} 25-\mathrm{WJ}$ is relatively lower. The results showed that the potential difference was about $97.3 \mathrm{mV}$ on average during the ages of 35 and 84 days. In contrast, the corrosion current density measured on the N25-WJ specimens were marginal and there is a slight possibility that corrosion of steel bars in the specimens occurred. Thus, the possibility of corrosion based on the results of half-cell potentials are not consistent with those of corrosion current density measured by the polarization resistance.

In principle, the half-cell potential of steel bars is lowered on condition that limiting current density is decreased owing to the reduction of oxygen concentrations which react in the cathodic polarization processes. In addition to the immersion tests using mortar specimens, polarization tests were carried out on the steel elements immersed in aqueous solution in this study. The set-up is similar to that used for the mortar specimens as shown in Fig. 5. The aqueous solution was prepared using distilled water and a culture solution of bacillus subtilis (natto). This was intentional to investigate the effect of dissolved oxygen concentrations on the cathodic reactions.

Fig. 6 shows the results of cathodic polarization

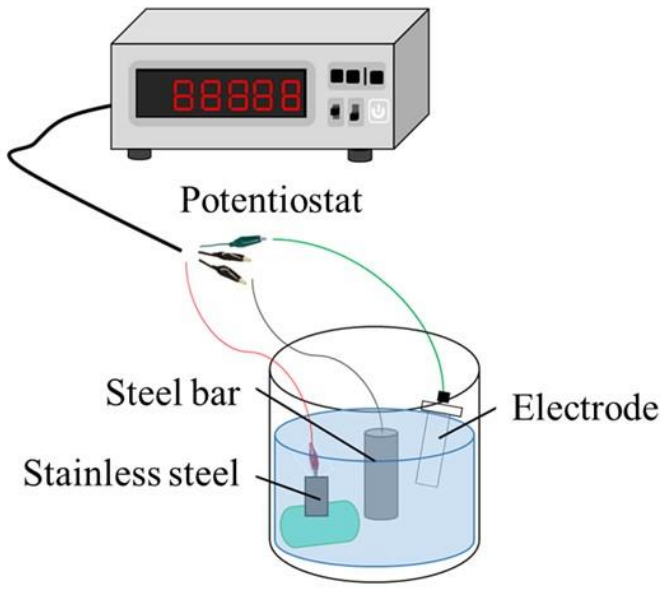

Fig. 5. Polarization measurements on round steel bar

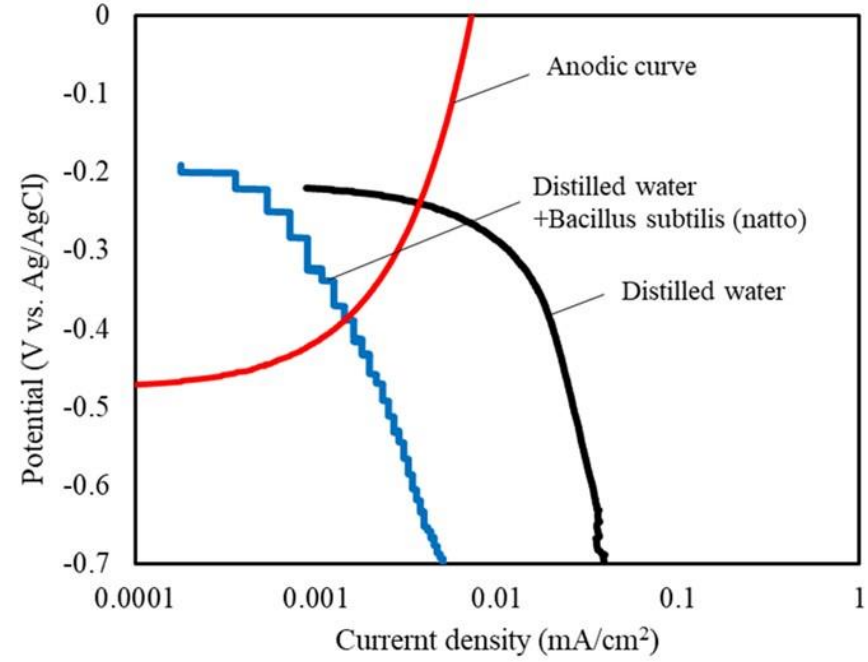

Fig. 6. Cathodic/Anodic polarization curves in aqueous solution 
curves in the cases of steel bars immersed in distilled water and the culture solution respectively. For illustration, the anodic polarization curve when corrosion occurs is also depicted in the figure. As can be seen, the cathodic polarization curve tends to shift on the left side in the case of the culture solution of bacillus subtilis (natto). This is attributed to the smaller limiting current density caused by the reduced availability of oxygen on the cathodic reactions in the culture solution of bacillus subtilis (natto). This led to lower half-cell potential at the cross point of two polarization curves in principle. Thus. The results suggest that dissolved oxygen concentration affects the half-cell potential measurements which is illustrated by the more negative potentials observed until the age of 91 days as mentioned previously. Thus, the lower potentials were found to show the evidence of decreased oxygen concentrations associated with the aerobic metabolic process by the bacillus subtilis (natto) mixed in the mortar specimens during the periods.

\subsection{Results of oxygen permeability}

Fig. 7 shows the results of rate of oxygen permeability measured at the ages of 91 and 182 days. It should be noted that the results of N50 mixtures were also included in order to show the variations during the ages. In the case of steel element $A$, the results of the rate of oxygen permeability are plotted in the vicinity of the line $(y=x)$. Although the permeation front of water could reach to the depth of steel bars embedded in the mortar specimens, the metabolic activity is likely to take place under wet conditions. Thus, the rate of oxygen permeability is generally lower compared to those observed in other steel elements. In addition, the variations during the time intervals were smaller under the saturated conditions. In contrast, as can be seen in the figures, the rate of oxygen permeability is generally higher in the cases of steel element B and C, which could be attributed to the dry environment in which moisture is evaporated from the pores and the activity of bacillus subtilis could be limited in part. This led to larger variations observed in the rate of oxygen permeability in those locations. Based on the results and discussion mentioned above, the effect of the presence of bacillus subtilis (natto) is most pronounced under the saturated conditions.

Fig. 8 and Fig. 9 show the rate of oxygen permeability measured on the steel element $A$ at the ages of 28 and 91 days. In the case of $\mathrm{N} 25-\mathrm{WJ}$, the rate of oxygen permeability is decreased by $28.6 \%$ and $48.9 \%$ at the ages of 28 and 91 days respectively. Based on the results obtained, the rate of oxygen permeability is clearly decreased from the age of 28 days to 91 days, which is consistent with the results reported by Kawaai et al., (2018). The results suggest that the consumption of dissolved oxygen associated with the metabolic processes of bacillus subtilis (natto) could contribute to the reduction of rate of oxygen permeability on the cathodic reactions. In addition, the lowered half-cell potentials observed especially until the age of 91 days could be attributed to the reduction of dissolved oxygen
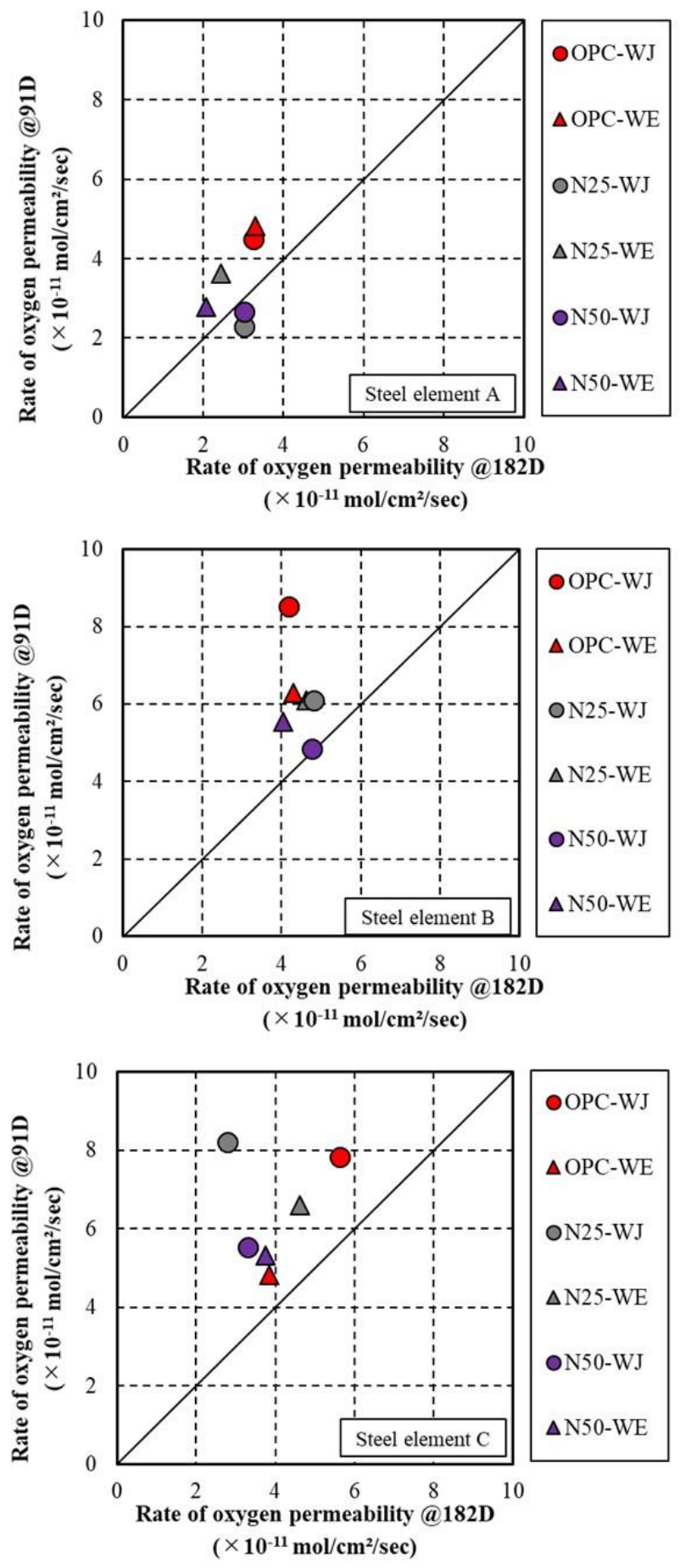

Fig. 7. Rate of oxygen permeability (Steel element A, B and C@91 and 182 days)

concentrations which can be illustrated by the changes of cathodic polarization curves. This needs further research especially for the interpretation of possibility of corrosion based on the half-cell potentials e.g. ASTM C 876.

\section{Summary}

The rate of oxygen permeability is clearly decreased from the age of 28 days to 91 days in the presence of bacillus subtilis (natto) under the saturated conditions. In addition, there is a good possibility that the half-cell 


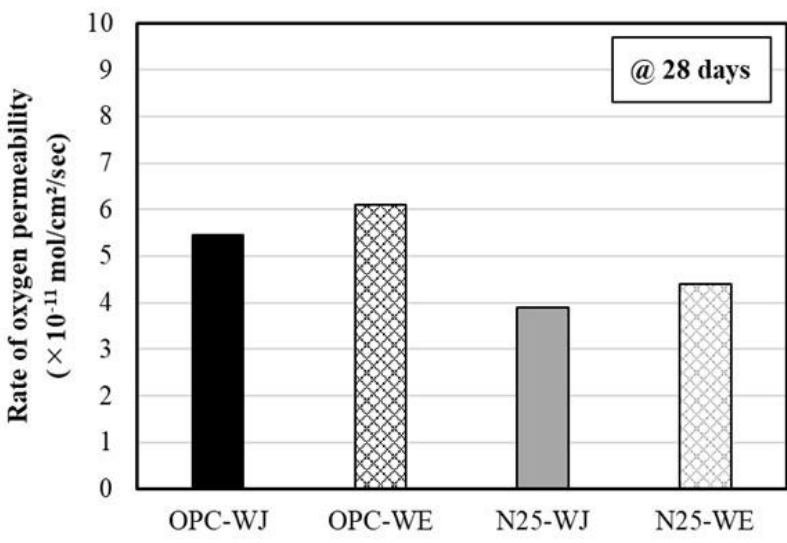

potential is lowered owing to reduced availability of dissolved oxygen concentrations in the presence of bacillus subtilis (natto), which can be illustrated by the changes of cathodic polarization curves. The lower potentials were found to show the evidence of decreased oxygen concentrations associated with the aerobic metabolic process by the bacillus subtilis (natto) mixed in the mortar specimens during the periods. The results suggest that the possibility of corrosion should be assessed taking into consideration other electro-chemical measurements e.g. polarization resistance not only based on the half-cell potentials.

Financial support by Grant-in-Aid for Young Scientists (A), Grant No. 17H04933 and ueda memorial foundation is gratefully acknowledged.

\section{References}

1. ASTM C876-91 R99, "Standard test method for halfcell potentials of uncoated reinforcing steel in concrete", ASTM International, West Conshohocken, PA. (1991)

2. I. Ujike, F. Kubo, K. Kawaai, and S. Okazaki, "Influencing Factors Affecting Microbial Metabolic

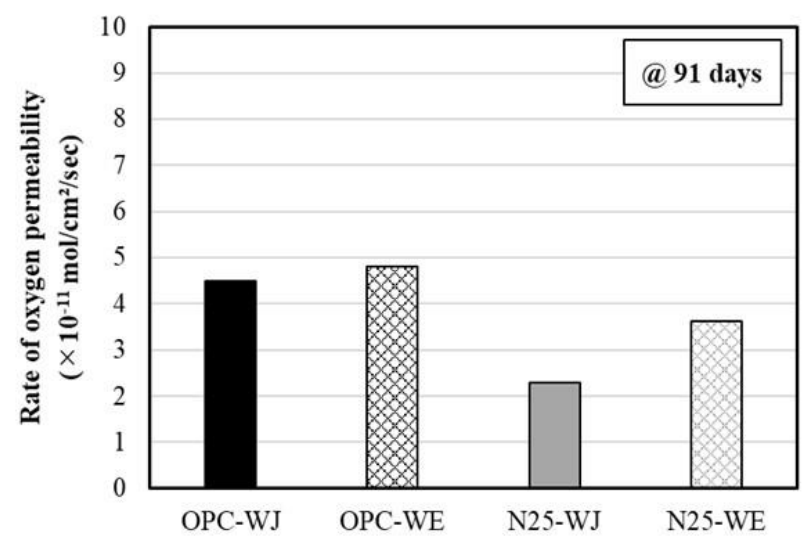

Processes of Bio Materials Used for Leakage Repairs", Concrete Solutions $5^{\text {th }}$ International Conference on Concrete Repair - Grantham et al. (Eds), CRC Press, pp. 127-133 (2014)

3. JIS R 5210, Portland cement, (2009)

4. K. Kawaai, I. Ujike, S. Fujioka, T. Nishida, and A. Saito, "Cathodic Polarization Properties of Steel Bars in Concrete Mixed with Aerobic Microorganism", Final Conference of RILEM TC 253-MCI, pp.259266 (2018)

5. RILEM technical committee, "Recommendation of RILEM TC 154-EMC: 'Electrochemical T3echniques for Measuring Metallic Corrosion'", Materials and Structures, Vol.36, pp. 461-471 (2003)

6. S. Nagataki., N. Otsuki., A. Moriwake, and S. Miyazato, "The experimental study on Corrosion Mechanism of Reinforced Concrete at Local Repair Part", Journal of Materials, Concrete Structures and Pavements", JSCE, 544(32), pp. 109-119 (1996)

7. V. Wiktor, and H.M., Jonkers, "Field Performance of Bacteria-Based Repair System: Pilot Study in a Parking Garage", Case Studies in Construction Materials, Vol. 2, pp. 11-17 (2015) 